TRANSACTIONS OF THE

AMERICAN MATHEMATICAL SOCIETY

Volume 355, Number 6, Pages 2569-2584

S 0002-9947(03)03170-2

Article electronically published on January 29, 2003

\title{
FIXED POINTS OF COMMUTING HOLOMORPHIC MAPPINGS OTHER THAN THE WOLFF POINT
}

\author{
FILIPPO BRACCI
}

\begin{abstract}
Let $\Delta$ be the unit disc of $\mathbb{C}$ and let $f, g \in \operatorname{Hol}(\Delta, \Delta)$ be such that $f \circ g=g \circ f$. For $A>1$, let $\operatorname{Fix}_{A}(f):=\left\{p \in \partial \Delta \mid \lim _{r \rightarrow 1} f(r p)=\right.$ $\left.p, \lim _{r \rightarrow 1}\left|f^{\prime}(r p)\right| \leq A\right\}$. We study the behavior of $g$ on $\operatorname{Fix}_{A}(f)$. In particular, we prove that $g\left(\operatorname{Fix}_{A}(f)\right) \subseteq \operatorname{Fix}_{A}(f)$. As a consequence, besides conditions for $\operatorname{Fix}_{A}(f) \cap \operatorname{Fix}_{A}(g) \neq \emptyset$, we prove a conjecture of C. Cowen in case $f$ and $g$ are univalent mappings.
\end{abstract}

\section{INTRODUCTION}

Let $\Delta:=\{z \in \mathbb{C}:|z|<1\}$. Let $h: \Delta \rightarrow \mathbb{C}$ be holomorphic and $x \in \partial \Delta$. We say that $h$ has non-tangential limit $L \in \mathbb{C}$ at $x$ if $\lim _{k \rightarrow \infty} h\left(z_{k}\right)=L$ for any sequence $\left\{z_{k}\right\} \subset \Delta$ such that $z_{k} \rightarrow x$ non-tangentially. For short we write either

$$
\mathrm{K}-\lim _{z \rightarrow x} h(z)=L
$$

or $h(x)=L$. The use of this notation should not be confusing, since we do not assume the maps to be continuous up to the boundary.

By the Wolff-Denjoy Theorem, if $f \in \operatorname{Hol}(\Delta, \Delta), f \neq I d$, there exists one distinguished point $\tau_{f} \in \bar{\Delta}$, the Wolff point of $f$, such that $f\left(\tau_{f}\right)=\tau_{f}$ and $\left|f^{\prime}\left(\tau_{f}\right)\right| \leq 1$. Moreover, if $\tau_{f} \in \partial \Delta$, then $0<f^{\prime}\left(\tau_{f}\right) \leq 1$.

If $g \in \operatorname{Hol}(\Delta, \Delta), g \neq I d$, and $f \circ g=g \circ f$, then Behan [Be] - generalizing a previous result of Shields Shi] for the case $f, g$ continuous up to $\partial \Delta$-proved that $f$ and $g$ have the same Wolff point unless they are two hyperbolic automorphisms. A consequence of this is that whenever $f, g \in \operatorname{Hol}(\Delta, \Delta)$ commute, then they must share a fixed point (in the sense of non-tangential limit if this point is on the boundary). This result has been generalized to domains in several variables by the author (see [Br1], Br2]), proving that a generic characteristic of commuting holomorphic mappings is that of sharing a "fixed point" in the closure of the domain.

By the Schwarz Lemma $f$ has at most one fixed point in $\Delta$ (provided $f \neq I d$ ). However, $f$ could have more than one fixed point (as non-tangential limit) at the boundary. Let us denote by $\operatorname{Fix}(f)$ the set of fixed points of $f$, i.e.,

$$
\operatorname{Fix}(f):=\{p \in \partial \Delta: f(p)=p\} \cup\left\{\tau_{f}\right\} .
$$

Received by the editors April 1, 2001.

2000 Mathematics Subject Classification. Primary 30D05; Secondary 30C80, 30E25, 47B33.

Key words and phrases. Fixed points; Wolff point; commuting mappings.

Partially supported by Progetto MURST di Rilevante Interesse Nazionale Proprietà geometriche delle varietà reali e complesse and GNSAGA. 
A natural question posed by C. Cowen [Co2] in 1984 was whether $g$ must have these points fixed as well. Simple examples show that this is not the case:

Example 1.1. Let $f(z)=(z)^{2}$ and $g(z)=(z)^{3}$ (here $(z)^{2}=z \cdot z,(z)^{3}=z \cdot z \cdot z$ ). Then the point -1 is fixed for $g$ but $f(-1)=1$. Note however that $f(-1)$ is a fixed point for $g$.

The conclusion of the previous example is not surprising, since, for $g, f$ continuous up to $\partial \Delta$, it follows easily that $g(\operatorname{Fix}(f)) \subset \operatorname{Fix}(f)$. Cowen himself provided an even worse example in which there exists a point $p \in \operatorname{Fix}(f)\left(\right.$ with $\left.f^{\prime}(p)<\infty\right)$ such that $g(p)$ is the Wolff point of $f$ (see Example 4.12 in [Co2], see also Example 4.3 below).

Notwithstanding such examples, Cowen conjectured the following:

(1) if the Wolff point $\tau_{f}$ of $f$ is on the boundary and $f^{\prime}\left(\tau_{f}\right)<1$, then for any $g \in \operatorname{Hol}(\Delta, \Delta)$ such that $f \circ g=g \circ f$ we have $\operatorname{Fix}(f)=\operatorname{Fix}(g)$;

(2) if the Wolff point of $f$ belongs to $\Delta, f \notin \operatorname{Aut}(\Delta)$, and $f^{\prime}\left(\tau_{f}\right) \neq 0$, then there exists $m \in \mathbb{N}$ such that for any $g \in \operatorname{Hol}(\Delta, \Delta), g \notin \operatorname{Aut}(\Delta)$ and $f \circ g=g \circ f$, if we set $g^{m}:=g^{m-1} \circ g, g^{1}=g$, it follows that $\operatorname{Fix}(f)=\operatorname{Fix}\left(g^{m}\right)$;

(3) if $f^{\prime}\left(\tau_{f}\right) \neq 0$, then for any $g \in \operatorname{Hol}(\Delta, \Delta)$ such that $f \circ g=g \circ f$ and $f, g$ have two fixed points in common we have $\operatorname{Fix}(f)=\operatorname{Fix}(g)$.

Aside from a lack of counterexamples, some geometrical intuitions and analogies with the continuous semigroup case (see Theorem 5.2 in Co1]), Cowen was able to support his idea with the following result, whose proof is based on the properties of linear fractional models (see [Co1]):

Proposition 1.2 (Cowen [Co2]). Suppose $f, g \in \operatorname{Hol}(\Delta, \Delta), f \circ g=g \circ f$. Moreover, suppose that $f^{\prime}$ is continuous on $\bar{\Delta}, \tau_{f} \in \partial \Delta, f^{\prime}\left(\tau_{f}\right)<1$, and there exists $p \in$ $\operatorname{Fix}(f), p \neq \tau_{f}$, such that $f\left(\bar{\Delta}-\left\{\tau_{f}, p\right\}\right) \subset \Delta$. Then $g(p)=p$.

After that we had no knowledge of any further step toward the (dis)proving of Cowen's conjecture(s). We became acquainted with some counterexamples to such conjectures constructed by means of Blaschke products due to Chalendar and Mortini [ChMo] only after a version of this work was under reviewing. The author thanks R. Tauraso for bringing the paper $\mathrm{ChMO}$ to his attention.

In this paper we restrict our attention to repelling boundary fixed points, giving some positive results in the direction of Cowen's conjectures. According to Poggi Corradini $[\mathrm{PC2}$, borrowing a word from discrete dynamics, we give the following definition.

Definition 1.3. Let $f \in \operatorname{Hol}(\Delta, \Delta)$ and $x \in \partial \Delta$. We say that $x$ is a boundary repelling fixed point, abbreviated BRFP, for $f$ if $f(x)=x$ and $1<f^{\prime}(x)<+\infty$. For $A>1$ we let

$$
\operatorname{Fix}_{A}(f):=\left\{x \in \partial \Delta: f(x)=x \text { and } f^{\prime}(x) \leq A\right\} .
$$

Note that, by the Julia-Wolff-Carathéodory Theorem (see Theorem 2.1), if $x \in$ $\partial \Delta$ is a fixed point for $f \in \operatorname{Hol}(\Delta, \Delta)$, then $f^{\prime}(x) \in(0,+\infty]$.

By definition the Wolff point $\tau_{f}$ of $f$ belongs to $\operatorname{Fix}_{A}(f)$ only if $\tau_{f} \in \partial \Delta$.

Our main result is the following.

Theorem 1.4. Let $f \in \operatorname{Hol}(\Delta, \Delta)$ (not the identity). Let $g \in \operatorname{Hol}(\Delta, \Delta)$ be nonconstant and such that $f \circ g=g \circ f$. Let $A>1$. Then $g\left(\operatorname{Fix}_{A}(f)\right) \subseteq \operatorname{Fix}_{A}(f)$, i.e., for any $p \in \operatorname{Fix}_{A}(f)$ the map $g$ has non-tangential limit $g(p) \in \operatorname{Fix}_{A}(f)$. 
The proof is based on the existence of backward iteration sequences for $f$ at each $p \in \operatorname{Fix}_{A}(f)$ due to Poggi Corradini (see Section 2), and some fine properties of "abstract backward iteration sequences", which we are going to prove.

After that we study the behavior of $g$ on $\operatorname{Fix}_{A}(f)$. If this set if finite - which is always the case if, e.g., $\tau_{f} \in \Delta$ or $\tau_{f} \in \partial \Delta$ and $f^{\prime}\left(\tau_{f}\right)<1$ - then $g$ has repelling cycles made of BRFP's for $f$. More explicitly:

Definition 1.5. Let $g \in \operatorname{Hol}(\Delta, \Delta), m \geq 1$ and $p_{0}, \ldots, p_{m-1} \in \partial \Delta, p_{j} \neq p_{l}$ for $l \neq j$. We say that $\left\{p_{0}, \ldots, p_{m-1}\right\}$ is a cycle for $g$ if $g\left(p_{j}\right)=p_{j+1}$ for $j=0, \ldots, m-2$ and $g\left(p_{m-1}\right)=p_{0}$. The number $m$ is called the length of the cycle. If in addition $1<\left|g^{\prime}\left(p_{j}\right)\right|<\infty$ for $j=0, \ldots, m-1$, we say that $\left\{p_{0}, \ldots, p_{m-1}\right\}$ is a repelling cycle for $g$.

Then the result is

Theorem 1.6. Let $f \in \operatorname{Hol}(\Delta, \Delta)$, not the identity. Let $g \in \operatorname{Hol}(\Delta, \Delta)$ be nonconstant and such that $f \circ g=g \circ f$. Let $A>1$ and suppose that $\operatorname{Fix}_{A}(f)$ is non-empty and finite. Then one and only one of the following may happen:

(1) either there exist $m \geq 1, p_{0}, \ldots, p_{m-1} \in \operatorname{Fix}_{A}(f), p_{l} \neq p_{j}$ for $l \neq j$, and $1<A^{\prime} \leq A$ such that $\left\{p_{0}, \ldots, p_{m-1}\right\}$ is a repelling cycle for $g$ and $f^{\prime}\left(p_{j}\right)=A^{\prime}$ for $j=0, \ldots, m-1$,

(2) or there exists $m \geq 1$ such that for any $p \in \operatorname{Fix}_{A}(f)$ it follows that $g^{m}(p)=$ $\tau_{f}$, where $\tau_{f} \in \partial \Delta$ is the Wolff point of $f$. In this case $\left(g^{m}\right)^{\prime}(p)=\infty$ for any $p \in \operatorname{Fix}_{A}(f)-\left\{\tau_{f}\right\}$.

Starting from Theorem 1.6, adding some extra hypotheses to $f$ and $g$, we get conditions for the existence of common fixed points other than the Wolff point (see Section 4). We also apply our results to the study of composition operators, giving a criterion for (non-)compactness of commuting composition operators (see Proposition 4.7). Finally, we prove Cowen's conjectures (1) and (2) and partially (3) in case $f, g$ are univalent on $\Delta$ and $f^{\prime}, g^{\prime}$ are continuous up to $\partial \Delta$.

Let us denote by $\operatorname{Hol}_{u}^{1}(\Delta, \Delta)$ the class of maps that are univalent on $\Delta$ and whose derivatives extend continuously up to the boundary, i.e.,

$\operatorname{Hol}_{u}^{1}(\Delta, \Delta):=\left\{h \in \operatorname{Hol}(\Delta, \Delta) \mid h\right.$ is univalent and $h^{\prime}$ is continuous on $\left.\bar{\Delta}\right\}$.

Note that if $f \in \operatorname{Hol}_{u}^{1}(\Delta, \Delta)$, then $f^{\prime}(a) \neq 0$ for any $a \in \bar{\Delta}$. Then:

Theorem 1.7. Suppose $f, g \in \operatorname{Hol}_{u}^{1}(\Delta, \Delta)$ and the Wolff point $\tau_{f}$ of $f$ is such that $\tau_{f} \in \partial \Delta$ and $f^{\prime}\left(\tau_{f}\right)<1$. If $f \circ g=g \circ f$, then $\operatorname{Fix}(f)=\operatorname{Fix}(g)$.

Theorem 1.8. Suppose $f \in \operatorname{Hol}_{u}^{1}(\Delta, \Delta)$ and the Wolff point $\tau_{f}$ of $f$ is such that $\tau_{f} \in \Delta$. Then there exists $m \in \mathbb{N}$ such that for any $g \in \operatorname{Hol}_{u}^{1}(\Delta, \Delta), g \notin \operatorname{Aut}(\Delta)$, if $f \circ g=g \circ f$, then $\operatorname{Fix}(f)=\operatorname{Fix}\left(g^{m}\right)$.

Proposition 1.9. Suppose $f, g \in \operatorname{Hol}_{u}^{1}(\Delta, \Delta)$ and $f \circ g=g \circ f$. Suppose $\tau_{f} \in \Delta$ and $\operatorname{Fix}(f) \cap \operatorname{Fix}(g)$ contains two points. Then $\operatorname{Fix}(f)=\operatorname{Fix}(g)$.

The author wants to warmly thank Pietro Poggi Corradini for many stimulating electronic discussions about topics related to this paper.

\section{Preliminary Results on backward iteration sequences}

We start by recalling the fundamental Julia-Wolff-Carathéodory Theorem (for a proof see, e.g., $\mathrm{Ab}$ or $\underline{\mathrm{Sha}}$ ). 
Theorem 2.1. Let $h \in \operatorname{Hol}(\Delta, \Delta)$ and $x \in \partial \Delta$. Let

$$
\alpha_{x}(f):=\liminf _{z \rightarrow x} \frac{1-|f(z)|}{1-|z|} .
$$

If $\alpha_{f}(x)<\infty$, then there exists $y \in \partial \Delta$ such that $f(x)=y$ and $f^{\prime}(x)=\bar{x} y \alpha_{f}(x)$. Moreover,

$$
f^{\prime}(x)=\liminf _{z \rightarrow x} \frac{1-|f(z)|}{1-|z|}=K-\lim _{z \rightarrow x} \frac{y-f(z)}{x-z} .
$$

For the reader's convenience we also report here some straightforward consequences of Theorem 4.1 and Lemma 8.2 of $[\mathrm{CoPO}$, which we are going to use later.

Theorem 2.2 (Cowen and Pommerenke [CoPo]$)$. Let $f \in \operatorname{Hol}(\Delta, \Delta)$ and let $\tau_{f} \in \bar{\Delta}$ be its Wolff point. Then

(1) If either $\tau_{f} \in \Delta$ or $\tau_{f} \in \partial \Delta$ and $f^{\prime}\left(\tau_{f}\right)<1$, then for any $A>1$ the set $\operatorname{Fix}_{A}(f)$ is finite.

(2) If $\tau_{f} \in \partial \Delta$ and $f^{\prime}\left(\tau_{f}\right)=1$, then for any $A>1$ the set $\operatorname{Fix}_{A}(f)$ can accumulate only at $\tau_{f}$.

(3) If $f$ is univalent, $p \in \partial \Delta$ and $\left|f^{\prime}(p)\right|<\infty$, then for any point $q \in \partial \Delta$, $q \neq p$, such that $f(q)=f(p)$, we have $\left|f^{\prime}(q)\right|=\infty$.

Remark 2.3. If $\left\{w_{n}\right\} \subset \Delta$ converges to $x$ radially, i.e., $\lim _{n \rightarrow \infty} \frac{1-\left|w_{n}\right|}{\left|1-w_{n}\right|}=1$, and $f \in \operatorname{Hol}(\Delta, \Delta)$ is such that $f(x)=x$, then

$$
\lim _{n \rightarrow 1} \frac{1-\left|f\left(w_{n}\right)\right|}{1-\left|w_{n}\right|}=f^{\prime}(x) .
$$

Indeed, $f^{\prime}(x) \leq \liminf _{n \rightarrow 1} \frac{1-\left|f\left(w_{n}\right)\right|}{1-\left|w_{n}\right|}$ by Theorem 2.1 Conversely,

$$
\frac{1-\left|f\left(w_{n}\right)\right|}{1-\left|w_{n}\right|} \leq \frac{\left|1-f\left(w_{n}\right)\right|}{\left|1-w_{n}\right|} \cdot \frac{\left|1-w_{n}\right|}{1-\left|w_{n}\right|},
$$

and again by Theorem 2.1 and the hypothesis on $\left\{w_{n}\right\}$, the right-hand side of the above equation tends to $f^{\prime}(x)$, giving the reverse inequality.

Let us indicate by $\omega(z, w)$ the hyperbolic distance on $\Delta$ (we refer the reader to, e.g., $\mathrm{Ab}$ for the definition and the properties of $\omega$ ). For $a \in \Delta$ the map $T_{a}(z):=\frac{a-z}{1-\bar{a} z}$ is an automorphism of $\Delta$, and $T_{a}(a)=0$. Then, for $z, w \in \Delta$,

$$
\omega(z, w)=\frac{1}{2} \log \frac{1+\left|T_{z}(w)\right|}{1-\left|T_{z}(w)\right|} .
$$

By the Schwarz-Pick Lemma, $\omega(f(z), f(w)) \leq \omega(z, w)$ for any $f \in \operatorname{Hol}(\Delta, \Delta)$, with equality at $z \neq w$ if and only if $f$ is an automorphism of $\Delta$.

Paraphrasing Poggi Corradini (see [PC2], we define a class of sequences associated to a holomorphic self-map of $\Delta$, which are going to be the main tool in the proof of our result.

Definition 2.4. Let $f \in \operatorname{Hol}(\Delta, \Delta)$. A sequence $\left\{w_{n}\right\} \subset \Delta$ is a backward iteration sequence if $f\left(w_{n+1}\right)=w_{n}$ and there exists a constant $C>0$ such that for all $n \in \mathbb{N}$,

$$
\omega\left(w_{n+1}, w_{n}\right)<C .
$$


Remark 2.5. We want to stress that in our definition a backward iteration sequence is a sequence whose steps are bounded in the hyperbolic metric (whereas in [PC2] this is not required).

Note that, by Lemma 2.1 in [PC2], if $f \in \operatorname{Hol}(\Delta, \Delta)$ and $x \in \partial \Delta$ is a BRFP for $f$, then a backward iteration sequence $\left\{w_{n}\right\}$ converging to $x$ such that for any $n \in \mathbb{N}$,

$$
\omega\left(w_{n}, w_{n+1}\right) \leq \frac{1}{2} \log f^{\prime}(x),
$$

actually converges radially to $x$.

The existence of backward iteration sequences at boundary repelling fixed points is guaranteed by the following theorem.

Theorem 2.6 (Poggi Corradini $[\mathrm{PC} 2]$ ). Let $f \in \operatorname{Hol}(\Delta, \Delta)$. Suppose $x \in \partial \Delta$ is a BRFP for $f$. Then there exists a backward iteration sequence $\left\{w_{n}\right\}$ for $f$ converging to $x$ and such that for any $n \in \mathbb{N}$,

$$
\omega\left(w_{n}, w_{n+1}\right) \leq \frac{1}{2} \log f^{\prime}(x) .
$$

Before going ahead we need an insight on abstract backward iteration sequences.

Lemma 2.7. Let $f \in \operatorname{Hol}(\Delta, \Delta)$. Let $\left\{w_{n}\right\}$ be a backward iteration sequence for $f$. Then one and only one of the following cases is possible:

(1) The map $f$ has Wolff point $\tau_{f} \in \Delta$, and $w_{n}=\tau_{f}$ for any $n \in \mathbb{N}$.

(2) There exists a unique point $x \in \partial \Delta$ such that $\lim _{n \rightarrow \infty} w_{n}=x$. Moreover, the point $x$ is a BRFP for $f$.

(3) The map $f$ has Wolff point $\tau_{f} \in \partial \Delta$, and the sequence $\left\{w_{n}\right\}$ is converging tangentially to $\tau_{f}$.

Proof. Suppose first that $\left\{w_{n}\right\}$ has an accumulation point $p \in \Delta$. Let $\tau_{f} \in \bar{\Delta}$ be the Wolff point of $f$. We claim that there exists a subsequence of $\left\{w_{n}\right\}$ that accumulates at $\tau_{f}$. If $p=\tau_{f}$, this is tautological. Suppose then that $p \neq \tau_{f}$. Let

$$
\mathcal{K}:=\left\{f^{m}\left(w_{n}\right)\right\}_{m \leq n}=\left\{w_{n-m}\right\}_{m \leq n}
$$

and

$$
d:=\inf _{q \in \overline{\mathcal{K}}}\left\{\left|q-\tau_{f}\right|\right\}
$$

Clearly $\tau_{f}$ is in the cluster set of $\left\{w_{n}\right\}$ if and only if $d=0$. Suppose $d>0$. Since $p \in \Delta$ and $\tau_{f}$ is the Wolff point of $f$, then there exists $t \in \mathbb{N}$ such that $\left|f^{t}(p)-\tau_{f}\right|<d$. But if $w_{n_{k}} \rightarrow p$, then $f^{t}\left(w_{n_{k}}\right) \rightarrow f^{t}(p)$, and therefore $f^{t}(p) \in \overline{\mathcal{K}}$, against the definition of $d$. Therefore there exists a subsequence $\left\{w_{l}\right\}$ of $\left\{w_{n}\right\}$ that converges to $\tau_{f}$. First suppose $\tau_{f} \in \partial \Delta$. Clearly in this case $f$ cannot be an automorphism of $\Delta$. Therefore, if we let $E_{0}$ be the horocycle (i.e., an open Euclidean disc contained in $\Delta$ and tangent to $\tau_{f}$ at $\partial \Delta$ ) such that $p \in \partial E_{0}$, let $E_{1}$ be the horocycle such that $f(p) \in \partial E_{1}$ and let $E_{2}$ be the horocycle such that $f^{2}(p) \in \partial E_{2}$, then by the Wolff Lemma (see, e.g., [Ab] or [Sha]) it follows that

$$
\overline{E_{2}} \subset E_{1} \subset \overline{E_{1}} \subset E_{0} .
$$

Hence there exist a neighborhood $U$ of $p$ and a neighborhood $V$ of $f^{2}(p)$ such that $\bar{U} \cap \overline{E_{1}}=\emptyset$ and $V \subset E_{1}$. Since $w_{n_{k}} \rightarrow p$, then $w_{n_{k}-2} \rightarrow f^{2}(p)$. Therefore there exist $l_{0}<l_{k}$ such that $w_{l_{0}} \in U$ and $w_{l_{k}} \in V$. By the Wolff Lemma, $f\left(E_{1}\right) \subset E_{1}$. 
But then $w_{l_{0}}=f^{l_{k}-l_{0}}\left(w_{l_{k}}\right) \in E_{1}$, a contradiction. Hence $\tau_{f} \in \Delta$. Therefore for any fixed $r \in \mathbb{N}$,

$$
\omega\left(\tau_{f}, w_{r}\right)=\omega\left(f\left(\tau_{f}\right), f\left(w_{r+1}\right)\right) \leq \omega\left(\tau_{f}, w_{r+1}\right) \leq \ldots \leq \omega\left(\tau_{f}, w_{m}\right),
$$

for any $m>r$. Since $w_{l} \rightarrow \tau_{f}$, this is possible if and only if $w_{n}=\tau_{f}$ for any $n$.

Suppose now that the cluster set of $\left\{w_{n}\right\}$ is contained in $\partial \Delta$. Using the definition of $\omega$, it is easy to see (or see, e.g., $\mathrm{Ab}$ ) that

$$
\frac{1}{2} \log f^{\prime}(x)=\liminf _{z \rightarrow x}[\omega(0, z)-\omega(0, f(z))] .
$$

Suppose that $\left\{w_{n_{k}}\right\}$ converges to $x \in \partial \Delta$. Then

$$
\left[\omega\left(0, w_{n_{k}}\right)-\omega\left(0, f\left(w_{n_{k}}\right)\right)\right] \leq \omega\left(w_{n_{k}}, f\left(w_{n_{k}}\right)\right)=\omega\left(w_{n_{k}}, w_{n_{k}-1}\right)<C<\infty .
$$

Therefore by Theorem $2.1 f$ has non-tangential limit at $x$, which is given by $y=$ $\lim _{k \rightarrow \infty} f\left(w_{n_{k}}\right)$ (this last assertion actually follows from a "metric version" of the Julia Lemma stated in more general domains than the disc, see [Br2]). Now

$$
\omega\left(w_{n_{k}}, f\left(w_{n_{k}}\right)\right)=\omega\left(w_{n_{k}}, w_{n_{k}-1}\right)<C,
$$

and therefore $\lim \sup _{k \rightarrow \infty} \omega\left(w_{n_{k}}, f\left(w_{n_{k}}\right)\right)<C$, which is possible only if $x=y$. Hence any point in the cluster set of $\left\{w_{n}\right\}$ is either a BRFP for $f$ or the Wolff point of $f$. Moreover, if $x$ is in the cluster set of $\left\{w_{n}\right\}$, then $f^{\prime}(x) \leq A$ where $A:=\exp (2 C)$, by (2.4). Suppose that $x$ is an isolated point in the cluster set of $\left\{w_{n}\right\}$. We claim that actually $\lim _{n \rightarrow \infty} w_{n}=x$. If not, there exist $\epsilon>0$ and a subsequence $\left\{w_{n_{l}}\right\}$ such that if we set $U_{\epsilon}:=\{z \in \Delta:|z-x|<\epsilon\}$, then $\left\{w_{n_{l}}\right\} \subset U_{\epsilon}$ but $w_{n_{l}+1} \notin \overline{U_{\epsilon}}$ for any $n_{l}$. But this contradicts (2.5). Hence either $\left\{w_{n}\right\}$ converges to a unique point, or the cluster set is a dense subset of an $\operatorname{arc}$ in $\partial \Delta$. In the last case there exist a point $x \in \operatorname{Fix}_{A}(f)$ and a sequence $\left\{x_{n}\right\} \subset \operatorname{Fix}_{A}(f)$ such that $x$ is not the Wolff point of $f$ and $x_{n} \rightarrow x$. This is impossible by Theorem 2.2.

Now suppose $\tau_{f} \in \partial \Delta$ is the limit of $\left\{w_{n}\right\}$. If $f$ is an automorphism, then clearly $f$ must be a parabolic automorphism, $w_{n}=f^{-n}\left(w_{0}\right)$ for some $w_{0} \in \Delta$, and $w_{n} \rightarrow \tau_{f}$ tangentially. Suppose $f$ is not an automorphism. Transfer to the right half-plane $H:=\{w \in \mathbb{C} \mid \operatorname{Re}(w)>0\}$ by means of the Cayley transform $C: \Delta \rightarrow H$ given by $C(z):=\frac{\tau_{f}+z}{\tau_{f}-z}$. Let $F:=C \circ f \circ C^{-1}$ and $z_{n}:=C \circ w_{n}$. Then $\left\{z_{n}\right\}$ is a backward iteration sequence for $F$ and $z_{n} \rightarrow \infty$, the Wolff point of $F$. By the (right half-plane) Wolff lemma, $\operatorname{Re}\left(z_{n}\right)=\operatorname{Re}\left(f\left(z_{n+1}\right)\right)>\operatorname{Re}\left(z_{n+1}\right)$, and hence $\left\{\operatorname{Re}\left(z_{n}\right)\right\}$ is a strictly decreasing sequence and thus has a limit $r \geq 0$. Hence $\left\{z_{n}\right\} \subset\{w \in \mathbb{C} \mid \operatorname{Re}(w)>r\}$ and $\lim _{n \rightarrow \infty} \operatorname{Re}\left(z_{n}\right)=r$. Interpreting this in the unit disc, we see that $w_{n} \rightarrow \tau_{f}$ tangentially either to $\partial E\left(1, \frac{1}{r}\right)$ if $r>0$ or to $\partial \Delta$ if $r=0$. In both cases $w_{n} \rightarrow \tau_{f}$ tangentially.

As the following examples show, a backward iteration sequence can converge to the Wolff point.

Example 2.8. If $f \in \operatorname{Hol}(\Delta, \Delta)$ is a parabolic automorphism, then $\left\{f^{-n}(0)\right\}$ is a backward iteration sequence for $f$ converging to its Wolff point.

Example 2.9. Let $\mathbb{H}:=\{w \in \mathbb{C}: \operatorname{Re}(w)>0\}$. Let $V:=\{x+i y \in \mathbb{H}: 1 \leq x \leq$ $2, y \leq 0\}$. Let $\sigma: \Delta \rightarrow \mathbb{H}-V$ be a Riemann mapping. Let $f(z):=\sigma^{-1}(\sigma(z)+i)$. Then $f \in \operatorname{Hol}(\Delta, \Delta)$ has Wolff point $\tau_{f}=\lim _{r \rightarrow+\infty} \sigma^{-1}(1+r i) \in \partial \Delta, f$ is not an automorphism, and $w_{n}:=\sigma^{-1}(3-n i)$ is easily seen to be a backward iteration sequence converging to the Wolff point of $f$. Note however that $f^{\prime}\left(\tau_{f}\right)=1$. 
It is not fortuitous that in the previous examples $f^{\prime}\left(\tau_{f}\right)=1$. Indeed, the following result holds.

Proposition 2.10. Let $f \in \operatorname{Hol}(\Delta, \Delta)$ and let $\tau_{f} \in \partial \Delta$ be its Wolff point. If $f^{\prime}\left(\tau_{f}\right)<1$, then there are no backward iteration sequences for $f$ converging to $\tau_{f}$.

The previous proposition was first proved by the author for univalent mappings in a preliminary version of this paper. After that, Prof. Poggi Corradini pursued a deep study of backward iteration sequences (see [PC3]), finding a completely different proof of such a result that worked for general mappings. Here we present our original proof, referring the reader to $\mathrm{PC}$ ] for the general case.

Proof of Proposition 2.10 for $f$ univalent. Suppose $\left\{w_{n}\right\}$ is a backward iteration sequence converging to $\tau_{f}$. Let $\lambda:=f^{\prime}\left(\tau_{f}\right)$. By Theorem 3.2 in Co1] and subsequent remarks, there exists a holomorphic map $\sigma: \Delta \rightarrow \mathbb{H}:=\{w \in \mathbb{C}: \operatorname{Re}(w)>0\}$ such that $\sigma$ is univalent on $\Delta$ and $\sigma \circ f(z)=\lambda \sigma(z)$. Let $\Phi(w):=\lambda w$ and $z_{n}:=\sigma\left(w_{n}\right)$. Then it is easy to see that $\Phi$ is a hyperbolic automorphism of $\mathbb{H}$ and $\left\{z_{n}\right\}$ is a backward iteration sequence for $\Phi$. Indeed,

$$
\Phi\left(z_{n}\right)=\Phi\left(\sigma\left(w_{n}\right)\right)=\sigma\left(f\left(w_{n}\right)\right)=\sigma\left(w_{n-1}\right)=z_{n-1}
$$

Therefore $z_{n}:=\Phi^{-n}\left(z_{0}\right)$ (from this it follows easily that $\left\{z_{n}\right\}$ has bounded hyperbolic steps, but we do not need this here). Note that $\lim _{n \rightarrow \infty} z_{n}=\infty$ and $\lim _{n \rightarrow \infty} \Phi^{n}\left(z_{0}\right)=0$. Now

$$
\begin{aligned}
\lim _{n \rightarrow \infty} \sigma^{-1}\left(z_{n}\right) & =\lim _{n \rightarrow \infty} w_{n}=\tau_{f}, \\
\lim _{n \rightarrow \infty} \sigma^{-1}\left(\Phi^{n}\left(z_{0}\right)\right) & =\lim _{n \rightarrow \infty} f^{n}\left(w_{0}\right)=\tau_{f} .
\end{aligned}
$$

Let $\gamma:(0,1) \rightarrow \mathbb{H}$ be a continuous curve joining 0 to $\infty$ and passing through $z_{n}$ and $\Phi^{n}\left(z_{0}\right)$ for any $n$. By Proposition 2.14 in $\left[\mathrm{Po}\right.$, then $\sigma^{-1}(\gamma(t))$ has distinct limits for $t \rightarrow 0$ and $t \rightarrow 1$. But this contradicts (2.6).

Theorem 2.11 (Poggi Corradini $[\mathrm{PC} 2]$ ). Let $f \in \operatorname{Hol}(\Delta, \Delta)$. Suppose 1 is a BRFP for $f$, and let $a:=\left(f^{\prime}(1)-1\right) /\left(f^{\prime}(1)+1\right)$ and $\Phi(z):=(z-a) /(1-a z)$. Then there exists $\sigma \in \operatorname{Hol}(\Delta, \Delta)$ such that

(1) $\mathrm{K}-\lim _{z \rightarrow 1} \sigma(z)=1$

(2) $\sigma \circ \Phi(z)=f \circ \sigma(z)$ for any $z \in \Delta$, and

(3) $\sigma$ is isogonal at 1, i.e. K- $\lim _{z \rightarrow 1} \arg \frac{1-\sigma(z)}{1-z}=0$.

Moreover, $\sigma$ is unique up to precomposition with a Möbius transformation $\Psi$ such that $\Psi \circ \Phi=\Phi \circ \Psi$.

We denote by $(f, \Phi, \sigma)$ the triple of functions as in Theorem 2.11, and call it a pre-model for $f$.

Remark 2.12. Let $(f, \Phi, \sigma)$ be a pre-model for $f$. If we set $a_{k}:=\Phi^{-k}(r)$ for $r \in(-1,1)$, then $\left\{a_{k}\right\}$ is a backward iteration sequence for $\Phi$ converging to 1 . Indeed, a simple computation shows that $\Phi$ is a hyperbolic automorphism of $\Delta$ with Wolff point -1 and repelling fixed point 1 -hence $\Phi^{-1}$ has Wolff point 1 . Moreover, since the automorphism $T_{r}(z):=(z-r) /(1-r z)$ commutes with $\Phi$, we 
have

$$
\begin{aligned}
\omega\left(a_{k}, a_{k+1}\right) & =\omega\left(\Phi^{-k}(r), \Phi^{-(k+1)}(r)\right)=\omega\left(r, \Phi^{-1}(r)\right) \\
& =\omega\left(T_{r}(r), T_{r} \circ \Phi^{-1}(r)\right)=\omega\left(T_{r}(r), \Phi^{-1} \circ T_{r}(r)\right) \\
& =\omega\left(0, \Phi^{-1}(0)\right)=\omega(0, a)=\frac{1}{2} \log f^{\prime}(1),
\end{aligned}
$$

and therefore the steps are bounded. Let $z_{k}:=\sigma\left(a_{k}\right)$. Then

$$
f\left(z_{k}\right)=f \circ \sigma\left(a_{k}\right)=\sigma \circ \Phi\left(a_{k}\right)=\sigma\left(a_{k-1}\right)=z_{k-1}
$$

and

$$
\omega\left(z_{k}, z_{k+1}\right)=\omega\left(\sigma\left(a_{k}\right), \sigma\left(a_{k+1}\right)\right) \leq \omega\left(a_{k}, a_{k+1}\right)=\frac{1}{2} \log f^{\prime}(1)<\infty .
$$

Therefore $\left\{z_{k}\right\}$ is a backward iteration sequence for $f$ converging to 1 .

What is really interesting for us is a converse of Remark 2.12

Proposition 2.13. Let $f \in \operatorname{Hol}(\Delta, \Delta)$ be such that 1 is a BRFP for $f$, and let $(f, \Phi, \sigma)$ be a pre-model for $f$ given by Theorem 2.11. Then, for any backward iteration sequence $\left\{w_{k}\right\}$ for $f$ converging to 1 such that for any $k \in \mathbb{N}$,

$$
\omega\left(w_{k}, w_{k+1}\right) \leq \frac{1}{2} \log f^{\prime}(1)
$$

there exists a backward iteration sequence $\left\{z_{k}\right\}$ for $\Phi$ converging to 1 such that $\sigma\left(z_{k}\right)=w_{k}$ for any $k \in \mathbb{N}$. Moreover, there exists $r \in(-1,1)$ such that $z_{k}=$ $\Phi^{-k}(r)$.

Proof. The proof of Theorem 2.11 (see [PC2]) is based on the existence of a backward iteration sequence for $f$-say $\left\{p_{k}\right\}$-such that $\omega\left(p_{k}, p_{k+1}\right) \leq \frac{1}{2} \log f^{\prime}(1)$ for any $k \in \mathbb{N}$. Once one has such a sequence, then $\sigma$ turns out to be a limit of the sequence $\left\{f^{n} \circ T_{n}\right\}$ where $T_{n}$ is an automorphism of $\Delta$ such that $T_{n}(0)=p_{n}$ for any $n$ and $\sigma\left(a_{k}\right)=p_{k}$ for $a_{k}=\Phi^{-k}(0)$. Therefore, given a backward iteration sequence $\left\{w_{n}\right\}$ satisfying (2.9), arguing as in the proof of Theorem 2.11, we can find another pre-model $(f, \Phi, \tilde{\sigma})$ for $f$ at 1 such that $\tilde{\sigma}\left(a_{k}\right)=w_{k}$. By the uniqueness statement in Theorem 2.11 it follows that there exists $\Psi \in \operatorname{Aut}(\Delta)$ commuting with $\Phi$ such that $\tilde{\sigma}=\sigma \circ \Psi$. Let $z_{k}:=\Psi\left(a_{k}\right)=\Phi^{-k}(\Psi(0))$. The sequence $\left\{z_{k}\right\}$ is a backward iteration sequence for $\Phi$ converging to 1 . Indeed, $z_{k}=\Psi\left(a_{k}\right) \rightarrow 1$ for $\Phi^{-1}$ is a hyperbolic automorphism with Wolff point 1. Moreover,

$$
\Phi\left(z_{k}\right)=\Phi \circ \Psi\left(a_{k}\right)=\Psi \circ \Phi\left(a_{k}\right)=\Psi\left(a_{k-1}\right)=z_{k-1},
$$

and by (2.7)

$$
\omega\left(z_{k}, z_{k+1}\right)=\omega\left(\Psi\left(a_{k}\right), \Psi\left(a_{k+1}\right)\right)=\omega\left(a_{k}, a_{k+1}\right)=\frac{1}{2} \log f^{\prime}(1)<\infty .
$$

Also

as desired.

$$
\sigma\left(z_{k}\right)=\tilde{\sigma} \circ \Psi^{-1}\left(\Psi\left(a_{k}\right)\right)=\tilde{\sigma}\left(a_{k}\right)=w_{k}
$$

Remark 2.14. Equations (2.3), (2.8) and Remark 2.3 imply that if $x \in \partial \Delta$ is a BRFP for $f$ and $\left\{w_{n}\right\}$ is a backward iteration sequence for $f$ converging to $x \in \partial \Delta$ with $\omega\left(w_{n}, w_{n+1}\right) \leq \frac{1}{2} \log f^{\prime}(x)$ for any $n \in \mathbb{N}$, then

$$
\frac{1}{2} \log f^{\prime}(x)=\lim _{n \rightarrow \infty}\left[\omega\left(0, w_{n}\right)-\omega\left(0, f\left(w_{n}\right)\right)\right] \leq \lim _{n \rightarrow \infty} \omega\left(w_{n}, w_{n+1}\right) \leq \frac{1}{2} \log f^{\prime}(x),
$$


and therefore,

$$
\frac{1}{2} \log f^{\prime}(x)=\lim _{n \rightarrow \infty} \omega\left(w_{n}, w_{n+1}\right) .
$$

Corollary 2.15. Let $f \in \operatorname{Hol}(\Delta, \Delta)$ and let $x \in \partial \Delta$ be a BRFP for $f$. If $\left\{w_{k}\right\}$ and $\left\{z_{k}\right\}$ are two backward iteration sequences for $f$ converging to 1 such that for any $k \in \mathbb{N}$,

$$
\omega\left(w_{k}, w_{k+1}\right) \leq \frac{1}{2} \log f^{\prime}(x)
$$

and

$$
\omega\left(z_{k}, z_{k+1}\right) \leq \frac{1}{2} \log f^{\prime}(x),
$$

then there exists $C>0$ such that for any $k \in \mathbb{N}$,

$$
\omega\left(w_{k}, z_{k}\right) \leq C .
$$

Proof. Up to conjugation we can suppose that $x=1$. Let $(f, \Phi, \sigma)$ be a pre-model for $f$ as given by Theorem 2.11. By Proposition 2.13 there exist two backward iteration sequences for $\Phi$ - say $\left\{a_{k}\right\}$ and $\left\{b_{k}\right\}$ - converging to 1 and such that $\sigma\left(a_{k}\right)=z_{k}$ and $\sigma\left(b_{k}\right)=w_{k}$. Therefore

$$
\omega\left(z_{k}, w_{k}\right)=\omega\left(\sigma\left(a_{k}\right), \sigma\left(b_{k}\right)\right) \leq \omega\left(a_{k}, b_{k}\right) .
$$

Now $\Phi\left(a_{k}\right)=a_{k-1}$ implies that $a_{k}=\Phi^{-1}\left(a_{k-1}\right)$. Therefore

$$
\omega\left(a_{k}, b_{k}\right)=\omega\left(\Phi^{-k}\left(a_{0}\right), \Phi^{-k}\left(b_{0}\right)\right)=\omega\left(a_{0}, b_{0}\right) .
$$

Combining (2.10) and (2.11), we get the result.

\section{BOUNDARY REPELLING FIXED POINTS}

In this section we prove Theorem 1.4 and, as a corollary, Theorem 1.6 The main idea is that if $x \in \partial \Delta$ is a BRFP for $f$ and $\left\{w_{n}\right\}$ is a backward iteration sequence converging to $x$, then $\left\{g\left(w_{n}\right)\right\}$ is a backward iteration sequence for $f$, which must converge either to a BRFP for $f$ or to the Wolff point of $f$. Then, using the Lindelöf theorem applied to a suitable curve, we show that $g$ has non-tangential limit at such a point. The results of the previous section then allow us to evaluate $f^{\prime}$ at such a point and to get the result.

Proof of Theorem 1.4 Up to conjugation we can suppose 1 is a BRFP for $f$. Let $\left\{w_{n}\right\}$ be a backward iteration sequence for $f$ converging to 1 (the existence is assured by Theorem [2.6) such that $\omega\left(w_{n}, w_{n+1}\right) \leq \frac{1}{2} \log f^{\prime}(1)$. Let $\tau_{f} \in \bar{\Delta}$ be the Wolff point of $f$. If $\tau_{f} \in \Delta$, we can choose $w_{0}$ in such a way that $g\left(w_{0}\right) \neq \tau_{f}$. Indeed, let $(f, \Phi, \sigma)$ be a pre-model for $f$ given by Theorem [2.11. Since $\sigma$ is non-constant, the image $\sigma((0,1 / 2))$ is a non-trivial curve in $\Delta$. The map $g$ being non-constant, there exists $w_{0} \in \sigma((0,1 / 2))$ such that $g\left(w_{0}\right) \neq \tau_{f}$. Let $z_{0} \in(0,1 / 2)$ be such that $\sigma\left(z_{0}\right)=w_{0}$. The sequence

$$
w_{n}:=\sigma\left(\Phi^{-n}\left(z_{0}\right)\right)
$$

is easily seen to be a backward iteration sequence for $f$ converging to 1 - just arguing as in the proof of Proposition 2.13 and (as in (2.7))

$$
\begin{aligned}
\omega\left(w_{n}, w_{n+1}\right) & =\omega\left(\sigma\left(\Phi^{-n}\left(z_{0}\right)\right), \sigma\left(\Phi^{-(n+1)}\left(z_{0}\right)\right)\right) \\
& \leq \omega\left(\Phi^{-n}\left(z_{0}\right), \Phi^{-(n+1)}\left(z_{0}\right)\right)=\frac{1}{2} \log f^{\prime}(1) .
\end{aligned}
$$

Moreover $g\left(w_{0}\right) \neq \tau_{f}$ by construction. Let

$$
w_{n}^{1}:=g\left(w_{n}\right)
$$


We claim that $\left\{w_{n}^{1}\right\}$ is a backward iteration sequence for $f$ with steps bounded by $\frac{1}{2} \log f^{\prime}(1)$. Indeed,

$$
f\left(w_{n}^{1}\right)=f \circ g\left(w_{n}\right)=g \circ f\left(w_{n}\right)=g\left(w_{n-1}\right)=w_{n-1}^{1}
$$

and

$$
\omega\left(w_{n}^{1}, w_{n+1}^{1}\right)=\omega\left(g\left(w_{n}\right), g\left(w_{n+1}\right)\right) \leq \omega\left(w_{n}, w_{n+1}\right) \leq \frac{1}{2} \log f^{\prime}(1) .
$$

By Lemma 2.7, either $\left\{w_{n}^{1}\right\}$ converges to a point $p \in \partial \Delta$ that is a BRFP for $f$ (or the Wolff point $\tau_{f}$ ) or $w_{n}^{1}=\tau_{f} \in \Delta$ for any $n \in \mathbb{N}$. But $w_{0}^{1}=g\left(w_{0}\right) \neq \tau_{f}$, and therefore the last possibility cannot occur. The sequence $\left\{w_{n}^{1}\right\}$ is a backward iteration sequence converging to $p \in \partial \Delta$. Moreover by (2.3),

$$
\begin{aligned}
\frac{1}{2} \log f^{\prime}(p) & \leq \lim _{n \rightarrow \infty}\left[\omega\left(0, w_{n}^{1}\right)-\omega\left(0, f\left(w_{n}^{1}\right)\right)\right] \leq \lim _{n \rightarrow \infty} \omega\left(w_{n}^{1}, f\left(w_{n}^{1}\right)\right) \\
& =\lim _{n \rightarrow \infty} \omega\left(g\left(w_{n}\right), g\left(f\left(w_{n}\right)\right)\right) \leq \lim _{n \rightarrow \infty} \omega\left(w_{n}, f\left(w_{n}\right)\right) \leq \frac{1}{2} \log f^{\prime}(1) .
\end{aligned}
$$

Therefore $f^{\prime}(p) \leq f^{\prime}(1)$. We have to show that $g$ has non-tangential limit $p$ at 1 . Let $(f, \Phi, \sigma)$ be a pre-model for $f$ at 1 given by Theorem 2.11 such that $\sigma\left(\Phi^{-n}(0)\right)=w_{n}$ (this is possible by Proposition 2.13). We are going to show that

$$
\lim _{t \rightarrow 1} g(\sigma(t))=p
$$

Once we have this, the Lindelöf Theorem (see, e.g., $[\mathrm{Ab}$ ) implies that $g$ has nontangential limit $p$ at 1 (recall that $\lim _{t \rightarrow 1} \sigma(t)=1$ ). Now

$$
[0,1]=\bigcup_{k \in \mathbb{N}} \Phi^{-k}([0, a]) .
$$

Let $\left\{t_{m}\right\} \subset(0,1)$ be such that $g\left(\sigma\left(t_{m}\right)\right) \rightarrow q \in \bar{\Delta}$. By (3.2), for any $m$ there exist $s_{m} \in[0, a)$ and $k_{m} \in \mathbb{N}$ such that $t_{m}=\Phi^{-k_{m}}\left(s_{m}\right)$. Therefore

$$
\begin{aligned}
\omega\left(g\left(\sigma\left(t_{m}\right)\right), g\left(w_{k_{m}}\right)\right) & \leq \omega\left(\sigma\left(t_{m}\right), w_{k_{m}}\right)=\omega\left(\sigma\left(\Phi^{-k_{m}}\left(s_{m}\right)\right), \sigma\left(\Phi^{-k_{m}}(0)\right)\right) \\
& \leq \omega\left(\Phi^{-k_{m}}\left(s_{m}\right), \Phi^{-k_{m}}(0)\right)=\omega\left(s_{m}, 0\right) \leq \omega(a, 0)<\infty .
\end{aligned}
$$

Hence, since $\left\{g\left(w_{n}\right)\right\}$ converges to $p$,

$$
q=\lim _{m \rightarrow \infty} g\left(\sigma\left(t_{m}\right)\right)=\lim _{m \rightarrow \infty} g\left(w_{m_{k}}\right)=p .
$$

This means that $\lim _{t \rightarrow 1} g(\sigma(t))=p$, as desired.

Remark 3.1. Suppose $f, g$ are as in the hypotheses of Theorem 1.4 If there are no backward iteration sequences converging to the Wolff point $\tau_{f}$ of $f$, then the proof of Theorem 1.6 shows that $g\left(\operatorname{Fix}_{A}(f)-\left\{\tau_{f}\right\}\right) \subset \operatorname{Fix}_{A}(f)-\left\{\tau_{f}\right\}$. This is always the case if either $\tau_{f} \in \Delta$, or $\tau_{f} \in \partial \Delta$ and $f^{\prime}\left(\tau_{f}\right)<1$, by Proposition 2.10

Remark 3.2. Let $f, g$ be as in the hypotheses of Theorem 1.4 and let $\tau_{f} \in \partial \Delta$ be the Wolff point of $f$. Note that if $p \in \partial \Delta$ is a BRFP for $f$ and $g(p)=\tau_{f}$, then $g^{\prime}(p)=\infty$. Indeed, if $\left\{w_{n}\right\}$ is a backward iteration sequence for $f$ given by Theorem 2.6] converging to $p$, then $w_{n} \rightarrow p$ radially, by Remark 2.5. If $\left|g^{\prime}(p)\right|<\infty$, then $g$ would be isogonal at $p$ and hence $\left\{g\left(w_{n}\right)\right\}$ would be a backward iteration sequence for $f$ converging to $\tau_{f}$ non-tangentially, in contradiction to Lemma 2.7

Theorem 1.6 will follow from the next result: 
Lemma 3.3. Let $f \in \operatorname{Hol}(\Delta, \Delta)$, not the identity. Let $g \in \operatorname{Hol}(\Delta, \Delta)$, not constant, be such that $f \circ g=g \circ f$. If there exist $l \in \mathbb{N}$ and $p \in \partial \Delta$ such that $p$ is a BRFP of $f$ and $g^{l}(p)=p$, then there exists a repelling cycle $\left\{p_{0}, \ldots, p_{m-1}\right\}$ for $g$ such that $m \leq l, p_{0}=p$, the points $p_{j}$ for $j=0, \ldots, m-1$ are BRFP's for $f$, and $f^{\prime}\left(p_{j}\right)=f^{\prime}(p)$ for $j=0, \ldots, m-1$.

Proof. Let $p_{0}:=p$. By Theorem 1.4 the map $g$ has non-tangential limit $p_{1} \in \partial \Delta$ at $p_{0}$, for some BRFP $p_{1}$ of $f$ and $f^{\prime}\left(p_{1}\right) \leq f^{\prime}\left(p_{0}\right)$ (the point $p_{1}$ cannot be the Wolff point of $f$, for otherwise it would be the Wolff point of $g$ as well and $g^{l}\left(p_{1}\right)=p_{1}$ for any $l \in \mathbb{N}$, or $f, g$ would be hyperbolic automorphisms and still this would result in a contradiction). By induction define $p_{j}:=g\left(p_{j-1}\right)$ for $j=1, \ldots, l$ (note that $\left.p_{l}=p_{0}\right)$. Let $m \leq l$ be the smallest positive integer such that $g^{m}\left(p_{0}\right)=p_{0}$. Therefore $\left\{p_{0}, \ldots, p_{m-1}\right\}$ are (different) BRFP's for $f$ such that $g\left(p_{j}\right)=p_{j+1}$ for $j=0, \ldots, m-1$ (here $\left.p_{m}=p_{0}\right)$. By Theorem 1.4 we get $f^{\prime}\left(p_{j}\right) \geq f^{\prime}\left(p_{j+1}\right)$ for $j=0, \ldots, m-1$; therefore $f^{\prime}\left(p_{j}\right)=f^{\prime}\left(p_{0}\right)$ for $j=1, \ldots, m-1$, as claimed. We want to show now that $p_{0}$ is a BRFP for $g^{m}$. Let $\left\{w_{n}\right\}$ be a backward iteration sequence for $f$ at $p_{0}$ such that $\omega\left(w_{n}, w_{n+1}\right) \leq \frac{1}{2} \log f^{\prime}\left(p_{0}\right)$. Then, arguing as in the proof of Theorem 1.4 it follows that $\left\{g^{m}\left(w_{n}\right)\right\}$ is a backward iteration sequence for $f$ converging to $p_{0}$ (since $g^{m}$ has non-tangential limit $p_{0}$ at $p_{0}$ and, by Remark 2.3, $w_{n} \rightarrow p_{0}$ non-tangentially) and

$$
\omega\left(g^{m}\left(w_{n}\right), g^{m}\left(w_{n+1}\right)\right) \leq \omega\left(w_{n}, w_{n+1}\right) \leq \frac{1}{2} \log f^{\prime}\left(p_{0}\right) .
$$

Then, by (2.3) and Corollary 2.15,

$$
\frac{1}{2} \log \left(g^{m}\right)^{\prime}\left(p_{0}\right) \leq \lim _{n \rightarrow \infty}\left[\omega\left(0, w_{n}\right)-\omega\left(0, g^{m}\left(w_{n}\right)\right)\right] \leq \lim _{n \rightarrow \infty} \omega\left(w_{n}, g^{m}\left(w_{n}\right)\right) \leq C .
$$

Therefore $g^{m}$ has non-tangential limit $p_{0}$ at $p_{0}$ and $\left(g^{m}\right)^{\prime}\left(p_{0}\right)<\infty$, i.e., $p_{0}$ is a BRFP for $g^{m}$. By Theorem [2.1] this implies that

$$
\lim _{r \rightarrow 1} \frac{1-\left|g^{m}\left(r p_{0}\right)\right|}{1-r}=\left(g^{m}\right)^{\prime}\left(p_{0}\right)<\infty .
$$

Therefore there exists $K>0$ such that for any $r \in(0,1)$,

$$
\begin{aligned}
K & >\frac{1-\left|g^{m}\left(r p_{0}\right)\right|}{1-r}=\frac{1-\left|g^{m}\left(r p_{0}\right)\right|}{1-\left|g\left(r p_{0}\right)\right|} \frac{1-\left|g\left(r p_{0}\right)\right|}{1-r} \\
& =\frac{1-\left|g^{m-1}\left(g\left(r p_{0}\right)\right)\right|}{1-\left|g\left(r p_{0}\right)\right|} \frac{1-\left|g\left(r p_{0}\right)\right|}{1-r} .
\end{aligned}
$$

Since by the Schwarz Lemma the two factors in the right-hand side of the above equation are bounded away from zero (see, e.g., Lemma 1.2.4 of [Ab]), then Theorem 2.1 implies that

$$
\left|g^{\prime}\left(p_{0}\right)\right| \leq \liminf _{r \rightarrow 1} \frac{1-\left|g\left(r p_{0}\right)\right|}{1-r}<\infty .
$$

By the same token and since $g\left(p_{0}\right)=p_{1},\left|\left(g^{m-1}\right)^{\prime}\left(p_{1}\right)\right|<\infty$. Repeating the above argument, we find that $\left|g^{\prime}\left(p_{j}\right)\right|<\infty$ for $j=1, \ldots, m-1$, as desired.

Now we come to the proof of Theorem 1.6

Proof of Theorem [1.6. By Theorem [1.4 if $q_{0} \in \mathrm{Fix}_{A}(f)$, then $g$ has non-tangential limit $q_{1}:=g\left(q_{0}\right)$ at $q_{0}$ and $q_{1} \in \operatorname{Fix}_{A}(f)$. Let $q_{2}=g\left(q_{1}\right)=g^{2}\left(q_{0}\right)$. Then $q_{2} \in$ $\operatorname{Fix}_{A}(f)$. Proceeding in this way, since $\operatorname{Fix}_{A}(f)$ is finite, we eventually either reach the Wolff point of $f$ or find $s<t \in \mathbb{N}$ such that $q_{t}=q_{s}$. 
In the last case we can choose $t$ in such a way that $q_{s+j} \neq q_{s}$ for $j=1, \ldots, t-s-1$. Let $m:=t-s$ and $p_{j}:=q_{s+j}$ for $j=0, \ldots, m-1$. Therefore $g$ is a permutation of $\left\{p_{0}, \ldots, p_{m-1}\right\}$, and the assertion comes from Lemma 3.3.

If every $p \in \operatorname{Fix}_{A}(f)$ is such that $g^{m_{p}}(p)=\tau_{f}$ for some $m_{p} \in \mathbb{N}$, since $\operatorname{Fix}_{A}(f)$ is finite, there exists $m \in \mathbb{N}$ such that $g^{m}(p)=\tau_{f}$ for any $p \in \operatorname{Fix}_{A}(f)$. Also $\left(g^{m}\right)^{\prime}(p)=\infty$ for any $p \in \operatorname{Fix}_{A}(f)$ by Remark 3.2

\section{EXAMPles, CONSEQUENCES AND APPLICATIONS}

We start with the following:

Proposition 4.1. Let $f, g \in \operatorname{Hol}(\Delta, \Delta)$ with $g$ non-constant and $f \circ g=g \circ f$. If the Wolff point $\tau_{f}$ of $f$ is contained in $\Delta$ and there exists $A>1$ such that $\operatorname{Fix}_{A}(f)$ contains only one point, say $p$, then $p$ is a BRFP for $g$.

Proof. By Theorem 1.6 there exists a repelling cycle $\left\{p_{0}, \ldots, p_{m-1}\right\}$ for $g$ made of elements of $\operatorname{Fix}_{A}(f)$. The only possibility is that $p_{j} \equiv p$ for $j=0, \ldots, m-1$, i.e., $m=1$, and therefore $p$ is a BRFP for $g$.

More generally, with the same proof (see also Remarks 3.1 and 3.2),

Proposition 4.2. Let $f, g \in \operatorname{Hol}(\Delta, \Delta)$ with $g$ non-constant and $f \circ g=g \circ f$. Let $\tau_{f} \in \bar{\Delta}$ be the Wolff point of $f$. Suppose that $\operatorname{Fix}_{A}(f)-\left\{\tau_{f}\right\}$ contains only one point, say $p$, for some $A>1$. If either there are no backward iteration sequences for $f$ converging to its Wolff point $\tau_{f}$ or $g^{\prime}(p)<\infty$, then $p$ is a BRFP for $g$.

The following example shows that the condition on the non-existence of backward iteration sequences converging to the Wolff point of $f$ cannot be deleted (see also Example 4.12 in [Co2] :

Example 4.3. Let $f$ and $\sigma$ be as in Example 2.9, Let $g(z):=\sigma^{-1}(\sigma(z)+2)$. Then $f \circ g=g \circ f$ and $g\left(\tau_{f}\right)=\tau_{f}$. However, $f$ fixes the point $x:=\lim _{r \rightarrow-\infty} \sigma^{-1}(1 / 2+r i)$ and $f^{\prime}(x)<\infty$, but $g(x)=\tau_{f}$. Note that $g^{\prime}(x)=\infty$.

Another consequence is the following:

Proposition 4.4. Let $f \in \operatorname{Hol}(\Delta, \Delta)$, not the identity, be such that its Wolff point $\tau_{f}$ belongs either to $\Delta$ or to $\partial \Delta$ and $f^{\prime}\left(\tau_{f}\right)<1$. Let $g \in \operatorname{Hol}(\Delta, \Delta)$ be such that $f \circ g=g \circ f$ and $g$ is non-constant. Suppose there exists $A>1$ such that $\operatorname{Fix}_{A}(f)$ is non-empty. Then there exists $m \geq 1$ such that $f$ and $g^{m}$ have (at least) $m$ common BRFP's.

Proof. By Theorem $2.2 \operatorname{Fix}_{A}(f)$ has a finite number of points. Therefore we can apply Theorem [1.6 to get a repelling cycle $\left\{p_{0}, \ldots, p_{m-1}\right\}$ for $g$ made of BRFP's for $f$ (see Remark 3.1). Then $g^{m}$ fixes each point of such a cycle.

The positive integer $m$ in Proposition 4.4 could be $>1$, as the following example shows:

Example 4.5. Let $p_{j}:=e^{2 j \pi i / 3}$ for $j=0,1,2$. For $j=0,1,2$ let $E_{j} \subset \Delta$ be the open disc of radius $1 / 2$ tangent to $\partial \Delta$ at $p_{j}$. Let $G:=\bigcup_{j=0}^{3} E_{j}$. The region $G$ is simply connected. Let $f: \Delta \rightarrow G$ be the Riemann mapping that fixes $p_{0}, p_{1}, p_{2}$ (see Theorem 6 on p. 46 in [Go]). Let $g(z):=e^{2 \pi i / 3} z$. Clearly $f \circ g=g \circ f$, the map $g$ has a repelling cycle made of $\left\{p_{0}, p_{1}, p_{2}\right\}$, and $g^{3}=I d$. 
In a sense the previous example shows that Cowen's conjecture (2) would not hold if $g$ is allowed to be an automorphism of $\Delta$.

Next we show that Theorem [1.6] does not hold if $\operatorname{Fix}_{A}(f)$ is infinite (and this may happen only if $f$ has Wolff point $\tau_{f}$ on the boundary and $f^{\prime}\left(\tau_{f}\right)=1$ ).

Example 4.6. Let $\mathbb{H}:=\{w \in \mathbb{C}: \operatorname{Re}(w)>0\}$. Let

$$
\begin{aligned}
V & :=\left\{x+i y \in \mathbb{H}:(x-1 / 2)^{2}+y^{2}<1 / 4\right\} \\
& \cup\{x+i y \in \mathbb{H}: x>1 / 2,-1 / 2<y<1 / 2\} .
\end{aligned}
$$

Let $\sigma:\{x+i y \in \mathbb{H}:-1 / 2<y<1 / 2\} \rightarrow V$ be a Riemann mapping such that $\sigma(0)=0$. Extend $\sigma$ by reflection to all of $\mathbb{H}$. Let $G:=\bigcup_{k \in \mathbb{Z}}(V+i k)$. Therefore $\sigma: \mathbb{H} \rightarrow G$ is univalent, $\sigma(k i)=k i$ and $\sigma^{\prime}(k i)=\sigma^{\prime}(0)<\infty$ for any $k \in \mathbb{Z}$. Let $C: \Delta \rightarrow \mathbb{H}$ be a Riemann mapping such that $C(1)=\infty$ and set $x_{k}:=C^{-1}(k i)$. Let $f(z):=C^{-1}(\sigma(C(z)))$ and $g(z):=C^{-1}(C(z)+i)$. By construction $f \circ g=g \circ f$. Moreover, $f\left(x_{k}\right)=x_{k}, f^{\prime}\left(x_{k}\right)=f^{\prime}\left(x_{0}\right)=A<\infty$ for any $k \in \mathbb{Z}$. Also $g, f$ have Wolff point at 1 , and $f^{\prime}(1)=g^{\prime}(1)=1$. Since $g\left(x_{k}\right)=x_{k+1}$, it follows that there is no $m \in \mathbb{N}$ such that $g^{m}(p)=\tau_{f}=1$ for any $p \in \operatorname{Fix}_{A}(f)$, and $g$ has no (finite) repelling cycle made of points of $\operatorname{Fix}_{A}(f)$.

An application of the previous results is about compactness of commuting composition operators. If $f \in \operatorname{Hol}(\Delta, \Delta)$ and $H^{2}(\Delta)$ is the Hardy space of square summable holomorphic functions, then $f$ induces an operator $C_{f}: H^{2}(\Delta) \rightarrow H^{2}(\Delta)$ given by $C_{f}(G):=G \circ f$ for any $G \in H^{2}(\Delta)$ (see, e.g., [Sha]). Given $g \in \operatorname{Hol}(\Delta, \Delta)$ such that $f \circ g=g \circ f$, it is clear that $C_{f} \circ C_{g}=C_{g} \circ C_{f}$. A natural question is whether $C_{f}$ compact implies $C_{g}$ compact. The answer is generally negative; just consider $f(z)=\frac{1}{2} z$ and $g(z)=z$ and apply the Compactness Criterion on p. 43 in Sha. However, we have the following:

Proposition 4.7. Let $f, g \in \operatorname{Hol}(\Delta, \Delta)$ be such that $f \circ g=g \circ f$. Suppose that Fix $_{A}(f)$ is non-empty for some $A>1$. Then $C_{f}$ and $C_{g}$ are not compact.

Proof. If $f$ has Wolff point $\tau_{f} \in \partial \Delta$, then by Behan's Theorem (see [Be]) $g$ has Wolff point $\tau_{g} \in \partial \Delta$. Since $f^{\prime}\left(\tau_{f}\right) \leq 1$ and $g^{\prime}\left(\tau_{g}\right) \leq 1$, then Theorem 2.1 and the Compactness Criterion (see the theorem on p. 43 of [Sha]) imply that $C_{f}, C_{g}$ are not compact. Suppose $\tau_{f} \in \Delta$. By Theorem [2.2, $\operatorname{Fix}_{A}(f)$ is finite. Hence by Theorem 1.6 there exists a point $p \in \operatorname{Fix}_{A}(f) \subset \partial \Delta$ such that $\left|f^{\prime}(p)\right|<\infty$ and $\left|g^{\prime}(p)\right|<\infty$. By Theorem 2.1 and the theorem on p. 43 of [Sha], this implies that $C_{f}$ and $C_{g}$ are not compact.

\section{The CASE OF Univalent FUnCtions}

In this section we restrict ourselves to the case when $f$ and $g$ are two univalent holomorphic self-maps of $\Delta$. If $f \circ g=g \circ f$ and $f$ is univalent, it is not true in general that $g$ is also univalent. However, whenever $f^{\prime}\left(\tau_{f}\right)<1$, then $g$ is univalent by Corollary 4.9 in [Co2]. The main point is to understand how a repelling cycle could be for univalent maps. For this we first need to introduce some notation. Let $\left\{p_{0}, \ldots, p_{m-1}\right\}$ be a cycle for $g \in \operatorname{Hol}(\Delta, \Delta)$. We give $p_{0}$ the number 0 . Then, moving counterclockwise on $\partial \Delta$ starting from $p_{0}$, we label with 1 the very next point $p_{j_{1}}$ of the cycle. We proceed counterclockwise to the next point $p_{j_{2}}$ of the cycle and number it with 2 , and so on. After introducing this labelling we have a way of seeing $g$ as a permutation of $\{0, \ldots, m-1\}$. For $l, k \in\{0, \ldots, m-1\}$ we write $g: l \mapsto k$ if $g\left(p_{j_{l}}\right)=p_{j_{k}}$. 
Lemma 5.1. Let $g \in \operatorname{Hol}(\Delta, \Delta)$ be univalent. Let $\left\{p_{0}, \ldots, p_{m-1}\right\}$ be a cycle (not necessarily a repelling cycle) for $g$ with length $m>1$. Then there exists $k \in$ $\{1, \ldots, m-1\}$ such that $g$ acts on the cycle $\left\{p_{0}, \ldots, p_{m-1}\right\}$ as the permutation

$$
j \mapsto j+k \bmod (m)
$$

for $j \in\{0, \ldots, m-1\}$.

Lemma 5.1 says that a univalent map has only a few ways of acting on its cycles. Moreover, it is not difficult to see that the $k$ in the lemma cannot be a divisor of $m$. Taking iterates, it is possible to construct examples with $k>1$. We wish to thank C. de Fabritiis for pointing out this fact.

Proof of Lemma 5.1. If $m=2,3$ the assertion is clear. Suppose $m>3$ and that the conclusion of the lemma is false. Up to relabelling the cycle we can suppose that $g: 0 \mapsto k$ and $g: 1 \mapsto k^{\prime}$ with $k^{\prime} \neq k+1$. First suppose $k=1$. Let $V$ be the arc on $\partial \Delta$ with end points $p_{1}$ and $p_{2}$ and which does not contain $p_{0}$. By hypothesis $p_{1}$ is associated to 1 and $p_{2}$ to some $l>2$. Therefore there exists some $p_{j_{s}} \in V$ with $j_{s} \neq 1,2$. Let $s, r \in\{3, \ldots, m-1\}$ be respectively the maximum and the minimum integer such that $p_{s}, p_{r} \in V$ (possibly $p_{s}=p_{r}$ ). Let $\gamma$ be the segment contained in $\Delta$ and joining $p_{0}$ to $p_{1}$, and let $\sigma$ be the segment in $\Delta$ that joins $p_{r-1}$ to $p_{s}$. By construction $\gamma \cap \sigma=\emptyset$. Now $g(\gamma)$ is a Jordan curve that joins $p_{1}$ to $p_{2}$ and whose closure disconnects $V$ from $\partial \Delta-V$. But $g(\sigma)$ is a Jordan curve that connects $p_{r} \in V$ to $p_{s+1} \notin V$, and therefore $g(\gamma)$ must intersect $g(\sigma)$ somewhere in $\Delta$, contradicting the fact that $g$ is univalent. Suppose now $k>1$. We are in the situation where $g: 0 \mapsto k$ but $g$ does not map 1 to $k+1$. Let $l \in\{0, \ldots, m-1\}$ be such that $g: 1 \mapsto k-1, g: 2 \mapsto k-2, \ldots, g: l \mapsto k-l$ and $g: l+1 \not \mapsto k-l-1$. Certainly $l<k$, because otherwise, if $k<m-1$, then $g$ would map $k$ to 0 , i.e., the cycle would contain $k<m-1$ elements; or, if $k=m-1$, then $g: 0 \mapsto k-1$ and $g: k-1 \mapsto 0$. Then $l<k$. Note that $g$ cannot map $l+1$ to $k-l+1$, since $k-(l-1)$ was to be mapped there. We can relabel the cycle in such a way that 0 corresponds to $p_{j_{l}}$. In this case $g: 0 \mapsto k^{\prime}=k-l, g: 1 \mapsto k_{1}$ with $k_{1} \neq k^{\prime}-1$ and $k_{1} \neq k^{\prime}+1$. If $k^{\prime}=1$, we can proceed as before. If $k^{\prime}>1$, we argue as follows. Let $q_{0}$ be the point of the cycle in position 0 and $q_{j_{1}}$ the one in position 1 . Let $\gamma$ be the segment in $\Delta$ joining $q_{0}$ to $q_{j_{1}}$, and $V$ the arc on $\partial \Delta$ with extremes $q_{1}:=g\left(q_{0}\right)$ and $q_{j_{1}+1}:=g\left(q_{j_{1}}\right)$ and which does not contain $q_{0}$. The arc $V$ minus the extremes contains more than one point of the cycle. If $q_{2}:=g\left(q_{1}\right) \in V$, let $r \in\{3, \ldots, m-1\}$ be the maximum such that $q_{r} \in V-\left\{q_{1}, q_{j_{1}+1}\right\}$. Then the segment $\sigma$ in $\Delta$ between $q_{1}$ and $q_{r}$ is such that $\gamma \cap \sigma=\emptyset$. But $g(\gamma) \cap g(\sigma) \neq \emptyset$ for $g(\gamma)$ has extremes $q_{1}, q_{j_{1}+1}$, and $g(\sigma)$ has extremes $q_{2} \in V$ and $q_{r+1} \notin V$. This contradicts the univalency of $g$. If $q_{2} \notin V$, then we choose $r \in\{3, \ldots, m-1\}$ to be the minimum such that $q_{r} \in V-\left\{q_{1}, q_{j_{1}+1}\right\}$. The segment $\sigma \subset \Delta$ that joins $p_{r-1}$ and $p_{1}$ is such that $\sigma \cap \gamma=\emptyset$ but $g(\sigma) \cap g(\gamma) \neq \emptyset$, again a contradiction.

Proposition 5.2. Let $g \in \operatorname{Hol}(\Delta, \Delta)$ be univalent and let $\left\{p_{0}, \ldots, p_{m-1}\right\}$ be a cycle for $g$. Let $\tau_{g}$ be the Wolff point of $g$. If $m>1$, then $\operatorname{Fix}(g)=\left\{\tau_{g}\right\}$ and $\tau_{g} \in \Delta$.

Proof. Suppose first $m>2$. By Lemma 5.1 the map $g$ can only act on the cycle as the permutation $j \mapsto j+k \bmod (m)$, for some fixed $k \in\{0, \ldots, m-1\}$. Suppose $p \in \partial \Delta$ is such that $g(p)=p$. Up to relabelling we can suppose that $p_{0}$ is the closest point of the cycle to $p$, moving from $p$ on $\partial \Delta$ counterclockwise. Let $\gamma$ be the segment in $\Delta$ joining $p$ to $p_{0}$, and $\sigma$ the segment between $p_{j_{1}}$-the point in 
position 1 - and $p_{m-1}$. Note that $p_{m-1}$ is in position $-k$, i.e., in position $k$ moving clockwise from $p_{0}$. By construction $\gamma \cap \sigma=\emptyset$. The closure of the Jordan curve $g(\gamma)$ connects $p$ to $p_{1}$. Let $V$ be the arc on $\partial \Delta$ with end points $p$ and $p_{1}$ that contains $p_{0}$. The closure of the curve $g(\sigma)$ joins $p_{0}$ with $p_{j_{1}+1}$. Since $p_{0} \in V$ (and it is not an extreme of $V$ ) and $p_{j_{1}+1} \notin V$, it follows that $g(\gamma) \cap g(\sigma) \neq \emptyset$, contradicting the fact that $g$ is univalent. Therefore $g$ cannot have any fixed point on $\partial \Delta$. This implies that the only fixed point of $g$ is its Wolff point $\tau_{g}$, and it must belong to $\Delta$.

We are left to consider the case $m=2$. Assume for a contradiction that $\tau_{g} \in \partial \Delta$. Let $\gamma_{j}$ be the segment between $\tau_{g}$ and $p_{j}, j=0,1$. Then $g\left(\gamma_{j}\right)$ is a curve joining $\tau_{g}$ to $p_{j}$. Since $g$ is isogonal at $\tau_{g}$, this implies that $g\left(\gamma_{0}\right) \cap g\left(\gamma_{1}\right) \cap \Delta \neq \emptyset$, which is impossible for $g$ is univalent. Therefore $\tau_{g} \in \Delta$. Suppose $p \in \partial \Delta$ is such that $g(p)=p$. Let $\sigma$ be the segment between $\tau_{g}$ and $p$ oriented by declaring $p-\tau_{g}$ a positive direction. Let $\gamma$ be a smooth oriented curve in $\Delta$ such that $\tau_{g} \in \gamma$, $\gamma \cap \sigma=\left\{\tau_{g}\right\}$. We also require that $p_{0}, p_{1}$ are the end points of $\gamma$ and $\gamma$ is nontangential to $\partial \Delta$ at $p_{0}, p_{1}$. Since $g$ preserves the orientation at $\tau_{g}$ (for $g^{\prime}\left(\tau_{g}\right) \neq 0$ ), then the curves $g(\gamma)$ and $g(\sigma)$ must intersect at $\tau_{g}$ with the same orientation as $\gamma \cap \sigma$. But this clearly implies that $g(\gamma)$ intersects $g(\sigma)$ at some point $q \in \Delta-\left\{\tau_{g}\right\}$, contradicting the fact that $g$ is univalent.

This is enough to prove Cowen's conjectures for univalent mappings, $C^{1}$ up to the boundary.

Proof of Theorem 1.7. If $\operatorname{Fix}(f)=\left\{\tau_{f}\right\}$ or $f$-and hence $g$-is a hyperbolic automorphism of $\Delta$, then the result follows from the Behan Theorem (see [Be]).

Suppose $\operatorname{Fix}(f)$ contains more than one point and $f$ is not an automorphism. Then $\tau_{f}=\tau_{g}$, and there exists $A>1$ such that $\operatorname{Fix}_{A}(f)$ is non-empty. Let

$$
A:=\max _{p \in \partial \Delta}\left|f^{\prime}(p)\right| \text {. }
$$

Certainly $1<A<\infty$. By Theorem 2.2 the set $\operatorname{Fix}_{A}(f)$ is finite and $\operatorname{Fix}_{A}(f)=$ Fix $(f)$. By Proposition $5.2, g$ may have only repelling cycles of length 1 . Therefore if we take any $p \in \operatorname{Fix}_{A}(f)$ and argue as in the proof of Lemma 3.3, we eventually find a finite sequence $p, g(p), \ldots, g^{m}(p)$ such that $g^{m}(p)$ is a fixed point of $g$ (note that if $p \neq \tau_{f}$, then $g^{m}(p) \neq \tau_{f}$ by Remark 3.1). We claim that $m=1$, i.e., $g(p)=p$. Suppose not. Let $q_{0}:=g^{m-1}(p)$ and $q_{1}:=g^{m}(p)$. Then $g\left(q_{0}\right)=g\left(q_{1}\right)=q_{1}$ and $\left|g^{\prime}\left(q_{0}\right)\right|<\infty,\left|g^{\prime}\left(q_{1}\right)\right|<\infty$. But this contradicts point (3) of Theorem [2.2.

Proof of Theorem 1.8. Suppose Fix $(f)$ contains more than one point (otherwise the result is trivial). Define $A$ as in equation (5.1). Then $\operatorname{Fix}_{A}(f)=\operatorname{Fix}(f)-\left\{\tau_{f}\right\}$ is a non-empty finite set, by Theorem 2.2 We partition $\operatorname{Fix}_{A}(f)$, giving the following equivalence relation: for $p, q \in \operatorname{Fix}_{A}(f)$ set $p \sim q$ if $f^{\prime}(p)=f^{\prime}(q)$. The number $N \in \mathbb{N}$ of equivalence classes is finite, and each class contains only a finite number of elements, say $a_{j} \in \mathbb{N}$ for $j=1, \ldots, N$. Let

$$
m:=\prod_{j=1}^{N} a_{j} !=\prod_{j=1}^{N}\left(a_{j}-1\right)\left(a_{j}-2\right) \cdots 1 .
$$

We claim that if $g$ is as in the hypotheses, then $\operatorname{Fix}\left(g^{m}\right)=\operatorname{Fix}(f)$. Clearly $\tau_{f}=\tau_{g}$. Let $p \in \operatorname{Fix}_{A}(f)$. Arguing as in the proof of Lemma 3.3 we can find a repelling cycle $\left\{g^{l}(p), \ldots, g^{l+m_{p}}(p)\right\}$ for $g$ of length $m_{p}+1 \geq 1$ made of BRFP's for $f$ such that $f^{\prime}\left(g^{l+j}(p)\right)=f^{\prime}\left(g^{l}(p)\right)$ for any $j=0, \ldots, m_{p}$. If $l>0$, let $q_{0}:=g^{l-1}(p)$ and 
$q_{1}=g^{l+m_{p}}(p)$. Then $q_{0} \neq q_{1}$ and $g\left(q_{0}\right)=g\left(q_{1}\right)$, which contradicts point (3) of Theorem 2.2 since $\left|g^{\prime}\left(q_{0}\right)\right|<\infty,\left|g^{\prime}\left(q_{1}\right)\right|<\infty$. Therefore $l=0$, i.e., each BRFP for $f$ is contained in a (unique) repelling cycle for $g$, and such a cycle is made of BRFP's where $f^{\prime}$ attains the same value. Hence the length of each cycle divides $m$, and so $g^{m}$ fixes every BRFP for $f$, as desired.

Finally:

Proof of Proposition 1.9. In case $\tau_{f} \in \Delta$, then $\tau_{g}=\tau_{f}$ and there exists $p \in \partial \Delta$, a BRFP for $f$ and $g$. Therefore by Proposition 5.2 the map $g$ may have only a repelling cycle of length 1 . The reasoning now goes exactly as in the proof of Theorem 1.7.

Remark 5.3. The previous proofs show that actually Theorems 1.7, 1.8 and Proposition 1.9 hold for a bigger class than $\operatorname{Hol}_{u}^{1}(\Delta, \Delta)$. Namely, they hold in the class of univalent maps on $\Delta$ whose derivatives are bounded on $\bar{\Delta}$. We thank M. Landucci for making this remark to us.

\section{REFERENCES}

[Ab] M. Abate, Iteration theory of holomorphic maps on taut manifolds. Mediterranean Press, Rende, Italy 1989. MR 92i:32032

[Be] D.F. Behan, Commuting analytic functions without fixed points. Proc. Amer. Math. Soc. 37 (1973), 114-120. MR 46:7492

[Br1] F. Bracci, Common fixed points of commuting holomorphic maps in the unit ball of $\mathbb{C}^{n}$. Proc. Amer. Math. Soc. 127, 4, (1999), 1133-1141. MR 99f:32034

[Br2] F. Bracci, Fixed points of commuting holomorphic maps without boundary regularity. Canad. Math. Bull. 43, 3, (2000), 294-303. MR 2001g:32039

[ChMo] I. Chalendar and R. Mortini, When do finite Blaschke products commute?. Bull. Australian Math. Soc., 64 (2001), 189-200. MR 2002i:30039

[Co1] C. C. Cowen, Iteration and the solution of functional equations for functions analytic in the unit disk. Trans. Amer. Math. Soc. 265 (1981), 69-95. MR 82i:30036

[Co2] C.C. Cowen, Commuting analytic functions. Trans. Amer. Math. Soc. 283,2, (1984), 685695. MR 85i:30054

[CoPo] C.C. Cowen and Ch. Pommerenke, Inequalities for the angular derivative of an analytic function in the unit disk. J. London Math. Soc. (2), 26 (1982), 271- 289. MR 84a:30006

[Go] G. M. Goluzin, Geometric theory of functions of a complex variable. Transl. of Math. Monographs, 26, Amer. Math. Soc. 1969. MR 40:308

[PC1] P. Poggi Corradini, Angular derivatives at boundary fixed points for self-maps of the disk. Proc. Amer. Math. Soc. 126, 6, (1998), 1697-1708. MR 98g:30049

[PC2] P. Poggi Corradini, Canonical conjugations at fixed points other than the Denjoy-Wolff point. Ann. Acad. Sci. Fenn. Math. 25 (2000), 2, 487-499. MR 2001f:30033

[PC3] P. Poggi Corradini, Backward sequences with bounded hyperbolic steps for analytic selfmaps of the disk. Revista Matematica Iberoamericana, to appear.

[Po] C. Pommerenke, Boundary behaviour of conformal maps. Springer-Verlag, New York, 1992. MR 95b:30008

[Sha] J.H. Shapiro, Composition operators and classical function theory. Springer-Verlag, New York, 1993. MR 94k:47049

[Shi] A. L. Shields, On fixed points of commuting analytic functions. Proc. Amer. Math. Soc. 15 (1964), 703-706. MR 29:2790

Dipartimento di Matematica, Università di Roma "Tor Vergata", Via della Ricerca SCIENTIFica 1, 00133 Roma, Italy

E-mail address: fbracci@mat.uniroma2.it 